J. Math. Soc. Japan

vol. 32 , No. 1,1980

\title{
Notes on infinitesimal variations of submanifolds
}

\author{
By Kentaro YANO
}

(Received April 1, 1978)

\section{$\S 0$. Introduction.}

In a previous paper [5], the present author studied variations of the metric tensor, the Christoffel symbols and the second fundamental tensors of submanifolds of a Riemannian manifold under infinitesimal variations of the submanifolds.

In this paper, we assume that submanifolds under consideration are compact and orientable and we obtain, using integral formulas, some global results on infinitesimal isometric, affine and conformal variations of the submanifolds.

\section{$\S 1$. Preliminaries [1].}

We consider an $m$-dimensional Riemannian manifold $M^{m}$ covered by a system of coordinate neighborhoods $\left\{U ; x^{h}\right\}$ and denote by $g_{j i}, \Gamma_{j i}^{h}$ and $\nabla_{j}$ the metric tensor, the Christoffel symbols formed with $g_{j i}$ and the operator of covariant differentiation with respect to $\Gamma_{j i}^{h}$ of $M^{m}$ respectively, where, here and in the sequel, the indices $h, i, j, k, \cdots$ run over the range $\left\{1^{\prime}, 2^{\prime}, \cdots, m^{\prime}\right\}$.

We then consider an $n$-dimensional compact orientable Riemannian manifold $M^{n}$ covered by a system of coordinate neighborhoods $\left\{V ; y^{a}\right\}$ and denote by $g_{c b}, \Gamma_{c b}^{a}, \nabla_{c}, K_{d c b}{ }^{a}$ and $K_{c b}$ the metric tensor, the Christoffel symbols formed with $g_{c b}$, the operator of covariant differentiation with respect to $\Gamma_{c b}^{a}$, the curvature tensor and the Ricci tensor of $M^{n}$ respectively, where, here and in the sequel, the indices $a, b, c, \cdots$ run over the range $\{1,2, \cdots, n\}$.

We assume that $M^{n}$ is isometrically immersed in $M^{m}$ by the immersion: $M^{n} \rightarrow M^{m}$ and represent the immersion by

$$
x^{h}=x^{h}\left(y^{a}\right) .
$$

Since the immersion is isometric, we have

$$
g_{c b}=B_{c}{ }^{j} B_{b}{ }^{i} g_{j i},
$$

where we have put $B_{c}{ }^{j}=\partial_{c} x^{j}\left(\partial_{c}=\partial / \partial y^{c}\right)$.

We can assume that $\left[B_{b}{ }^{h}\right]$ gives the positive orientation of $M^{n}$. 
We choose $m-n$ mutually orthogonal unit normals $C_{y}{ }^{n}$ to $M^{n}$, where, here and in the sequel, the indices $x, y, z$ run over the range $\{n+1, \cdots, m\}$. The metric tensor of the normal bundle of $M^{n}$ is given by

$$
g_{z y}=C_{z}^{j} C_{y}^{i} g_{j i} \text {. }
$$

Now, the equations of Gauss for $M^{n}$ are written as

$$
\nabla_{c} B_{b}^{n}=h_{c b}^{x} C_{x}^{h},
$$

where

$$
\nabla_{c} B_{b}{ }^{h}=\partial_{c} B_{b}{ }^{h}+\Gamma_{j i}^{h} B_{c}{ }^{j} B_{b}{ }^{i}-\Gamma_{c b}^{a} B_{a}{ }^{h}
$$

is the van der Waerden-Bortolotti covariant derivative of $B_{b}{ }^{h}$ and $h_{c b}{ }^{x}$ are components of the second fundamental tensor with respect to the normal $C_{x}{ }^{h}$.

On the other hand, the equations of Weingarten for $M^{n}$ are written as

$$
\nabla_{c} C_{y}{ }^{h}=-h_{c}^{a}{ }_{y} B_{a}{ }^{h}
$$

where

$$
\nabla_{c} C_{y}{ }^{h}=\partial_{c} C_{y}{ }^{h}+\Gamma_{j i}^{h} B_{c}{ }^{j} C_{y}{ }^{i}-\Gamma_{c y}^{x} C_{x}{ }^{h}
$$

is the van der Waerden-Bortolotti covariant derivative of $C_{y}{ }^{h}, \Gamma_{c y}^{x}$ being components of the linear connection induced in the normal bundle, that is,

$$
\Gamma_{c y}^{x}=\left(\partial_{c} C_{y}{ }^{h}+\Gamma_{j i}^{h} B_{c}{ }^{j} C_{y}{ }^{i}\right) C^{x}{ }_{h}
$$

and $C^{x}{ }_{n}=C_{y}{ }^{i} g^{y x} g_{i h}, g^{y x}$ being contravariant components of the metric tensor of the normal bundle of $M^{n}$ and

$$
h_{c y}^{a}=h_{c b}^{z} g^{b a} g_{z y},
$$

$g^{b a}$ being contravariant components of the metric tensor of $M^{n}$.

\section{§2. Infinitesimal variations [2] [3] [5].}

We now consider an infinitesimal variation of $M^{n}$ given by

$$
\bar{x}^{h}=x^{h}+\xi^{h}(y) \varepsilon,
$$

where $\xi^{h}(y)$ is a vector field defined along $M^{n}$ and $\varepsilon$ is an infinitesimal.

Under the infinitesimal variation (2.1) the vectors $B_{b}{ }^{n}$ tangent to $M^{n}$ are transformed into $\bar{B}_{b}{ }^{h}=\partial_{b} \bar{x}^{h}=B_{b}{ }^{h}+\partial_{b} \xi^{h} \varepsilon$ tangent to the deformed submanifold.

Carrying $\bar{B}_{0}{ }^{h}$ at $\left(\bar{x}^{h}\right)$ back to $\left(x^{h}\right)$ parallelly, we obtain

that is,

$$
\tilde{B}_{b}{ }^{h}=\bar{B}_{b}{ }^{h}+\Gamma_{j i}^{h}(x+\xi \varepsilon) \xi^{j} \bar{B}_{b}{ }^{i} \varepsilon,
$$




$$
\hat{B}_{b}{ }^{h}=B_{b}{ }^{h}+\left(\nabla_{b} \xi^{h}\right) \varepsilon,
$$

neglecting terms of order higher than one with respect to $\varepsilon$, where

$$
\nabla_{b} \xi^{h}=\partial_{b} \xi^{h}+\Gamma_{j i}^{h} B_{b}{ }^{j} \xi^{i} .
$$

In the sequel we always neglect the terms of order higher than one with respect to $\varepsilon$.

Thus putting $\delta B_{b}{ }^{h}=\widetilde{B}_{b}{ }^{h}-B_{b}{ }^{h}$, we have

$$
\delta B_{b}{ }^{h}=\left(\nabla_{b} \xi^{h}\right) \varepsilon .
$$

If we decompose $\xi^{h}$ as

$$
\xi^{h}=\xi^{a} B_{a}{ }^{h}+\xi^{x} C_{x}{ }^{n},
$$

equation (2.4) can be written as

$$
\delta B_{b}{ }^{h}=\left[\left(\nabla_{b} \xi^{a}-h_{b}{ }^{a}{ }_{x} \xi^{x}\right) B_{a}{ }^{h}+\left(\nabla_{b} \xi^{x}+h_{b a}{ }^{x} \xi^{a}\right) C_{x}{ }^{h}\right] \varepsilon .
$$

Thus, from (1.1) and (2.6), we find [5]

$$
\delta g_{c b}=\left(\nabla_{c} \xi_{b}+\nabla_{b} \xi_{c}-2 h_{c b x} \xi^{x}\right) \varepsilon,
$$

where $\xi_{b}=\xi^{c} g_{c b}$ and $h_{c b x}=h_{c b}{ }^{y} g_{y x}$.

From (2.7) we find [5]

$$
\delta g^{b a}=-\left(\nabla^{b} \xi^{a}+\nabla^{a} \xi^{b}-2 h^{b a}{ }_{x} \xi^{x}\right) \varepsilon,
$$

where $\nabla^{b}=g^{b a} \nabla_{a}$ and $h^{b a}{ }_{x}=h_{e d x} g^{e b} g^{d a}$.

When $\delta g_{c b}=0$, we say that the infinitesimal variation is isometric and when $\delta g_{c b}=2 \lambda g_{c b} \varepsilon, \lambda$ being a certain scalar, we say that the infinitesimal variation is conformal. If the variation is conformal and $\lambda$ is a constant, we say that the infinitesimal variation is homothetic.

From (2.7) we have

THEOREM A. [5] In order for an infinitesimal variation (2.1) of a submanifold to be isometric, it is necessary and sufficient that

$$
\nabla_{c} \xi_{b}+\nabla_{b} \xi_{c}-2 h_{c b x} \xi^{x}=0 \text {. }
$$

THEOREM B. [5] In order for an infinitesimal variation (2.1) of a submanifold to be conformal, it is necessary and sufficient that

$$
\nabla_{c} \xi_{b}+\nabla_{b} \xi_{c}-2 h_{c b x} \xi^{x}=2 \lambda g_{c b},
$$

$\lambda$ being a certain scalar.

Using (2.7) and (2.8), we calculate the infinitesimal variation of the 
Christoffel symbols

$$
\Gamma_{c b}^{a}=\frac{1}{2}\left(\partial_{c} g_{b e}+\partial_{b} g_{c e}-\partial_{e} g_{c b}\right) g^{e a}
$$

and obtain

$$
\delta \Gamma_{c b}^{a}=\frac{1}{2}\left[\nabla_{c}\left(\delta g_{b e}\right)+\nabla_{b}\left(\delta g_{c e}\right)-\nabla_{e}\left(\delta g_{c b}\right)\right] g^{e a},
$$

which, using (2.7), we car. write as

$$
\delta \Gamma_{c b}^{a}=\left[\nabla_{c} \nabla_{b} \xi^{a}+K_{d c b}{ }^{a} \xi^{d}-\nabla_{c}\left(h_{b}{ }^{a}{ }_{x} \xi^{x}\right)-\nabla_{b}\left(h_{c}{ }^{a} \xi^{x}\right)+\nabla^{a}\left(h_{c b x} \xi^{x}\right)\right] \varepsilon .
$$

If $\delta \Gamma_{c b}^{a}=0$, we say that the infinitesimal variation is affine and if $\delta \Gamma_{c b}^{a}$ $=\left(\delta_{c}^{a} p_{b}+\delta_{b}^{a} p_{c}\right) \varepsilon$ for some 1 -form $p_{b}$, we say that the variation is projective.

Thus we have

THEOREM C. [5] In order for an infinitesimal variation (2.1) of a submanifold to be affine, it is necessary and sufficient that

$$
\nabla_{c} \nabla_{b} \xi^{a}+K_{d c b}{ }^{a} \xi^{d}-\nabla_{c}\left(h_{b}{ }^{a}{ }_{x} \xi^{x}\right)-\nabla_{b}\left(h_{c}{ }^{a}{ }_{x} \xi^{x}\right)+\nabla^{a}\left(h_{c b x} \xi^{x}\right)=0 .
$$

Now we have the following integral formula [4]

$$
\int\left[\left(g^{c b} \nabla_{c} \nabla_{b} \xi^{a}+K_{d}^{a} \xi^{d}\right) \xi_{a}+\frac{1}{2}\left(\nabla_{c} \xi_{b}+\nabla_{b} \xi_{c}\right)\left(\nabla^{c} \xi^{b}+\nabla^{b} \xi^{c}\right)-\left(\nabla_{a} \xi^{a}\right)^{2}\right] d V=0,
$$

which is valid for an arbitrary vector field $\xi^{a}$ in a compact orientable Riemannian manifold $M^{n}, d V$ being the volume element of the manifold.

From (2.15), we can easily derive

$$
\begin{aligned}
& \int\left[\left\{g^{c b} \nabla_{c} \nabla_{b} \xi^{a}+K_{d}{ }^{a} \xi^{d}-2 \nabla^{c}\left(h_{c}{ }^{a}{ }_{x} \xi^{x}\right)+\nabla^{a}\left(h_{c}{ }^{c}{ }_{x} \xi^{x}\right)\right\} \xi_{a}\right. \\
&+ \frac{1}{2}\left(\nabla_{c} \xi_{b}+\nabla_{b} \xi_{c}-2 h_{c b y} \xi^{y}\right)\left(\nabla^{c} \xi^{b}+\nabla^{b} \xi^{c}-2 h^{c b}{ }_{x} \xi^{x}\right) \\
&+\left(\nabla_{c} \xi_{b}+\nabla_{b} \xi_{c}-2 h_{c b y} \xi^{y}\right) h^{c b}{ }_{x} \xi^{x} \\
&\left.-\left(\nabla_{c} \xi^{c}-h_{c}{ }_{x} \xi^{x}\right)\left(\nabla_{b} \xi^{b}\right)\right] d V=0,
\end{aligned}
$$

which is valid for arbitrary $\xi^{a}$ and $\xi^{x}$.

Now suppose that an infinitesimal variation (2.1) of the submanifold is isometric. Then since it is affine, we have (2.14), from which, we have, by transvection with $g^{c b}$,

$$
g^{c b} \nabla_{c} \nabla_{b} \xi^{a}+K_{d}{ }^{a} \xi^{d}-2 \nabla^{c}\left(h_{c}{ }^{a}{ }_{x} \xi^{x}\right)+\nabla^{a}\left(h_{c}{ }^{c}{ }_{x} \xi^{x}\right)=0 .
$$

We also obtain from (2.9), 


$$
\left(\nabla_{c} \xi_{b}+\nabla_{b} \xi_{c}-2 h_{c b y} \xi^{y}\right) h^{c b} \xi^{x}=0
$$

and

$$
\left(\nabla_{c} \xi^{c}-h_{c}{ }^{c} \xi^{x}\right)\left(\nabla_{b} \xi^{b}\right)=0 .
$$

Conversely if (2.17), (2.18) and (2.19) are satisfied, we have, from (2.16),

$$
\nabla_{c} \xi_{b}+\nabla_{b} \xi_{c}-2 h_{c b y} \xi^{y}=0
$$

which shows that the infinitesimal variation is an isometry. Thus we have

THEOREM D. [5] In order for an infinitesimal variation of a compact orientable submanifold of a Riemannian manifold to be isometric, it is necessary and sufficient that we have (2.17), (2.18) and (2.19).

\section{$\S 3$. Infinitesimal isometries.}

Suppose that the infinitesimal variation (2.1) is an isometry. Then we have (2.17), Thus substituting (2.17) into

we find

$$
\begin{aligned}
\frac{1}{2} \Delta\left(\xi^{a} \xi_{a}\right) & =\frac{1}{2} g^{c b} \nabla_{c} \nabla_{b}\left(\xi^{a} \xi_{a}\right) \\
& =\left(g^{c b} \nabla_{c} \nabla_{b} \xi^{a}\right) \xi_{a}+\left(\nabla^{c} \xi^{b}\right)\left(\nabla_{c} \xi_{b}\right),
\end{aligned}
$$

$$
\frac{1}{2} \Delta\left(\xi^{a} \xi_{a}\right)=-K_{c b} \xi^{c} \xi^{b}+\left\{2 \nabla^{c}\left(h_{c}{ }^{a}{ }_{x} \xi^{x}\right)-\nabla^{a}\left(h_{c}{ }^{c}{ }_{x} \xi^{x}\right)\right\} \xi_{a}+\left(\nabla^{c} \xi^{b}\right)\left(\nabla_{c} \xi_{b}\right),
$$

from which, by integration over $M^{n}$,

$$
0=\int\left[-K_{c b} \xi^{c} \xi^{b}-2 h^{c b}{ }_{x} \xi^{x} \nabla_{c} \xi_{b}+\left(h_{c}{ }^{c} \xi^{x}\right)\left(\nabla_{b} \xi^{b}\right)+\left(\nabla^{c} \xi^{b}\right)\left(\nabla_{c} \xi_{b}\right)\right] d V
$$

or

$$
\int\left[-K_{c b} \xi^{c} \xi^{b}-h_{y}^{c b} \xi^{y}\left(\nabla_{c} \xi_{b}+\nabla_{b} \xi_{c}\right)+\left(h_{c}^{c} x \xi^{x}\right)\left(\nabla_{b} \xi^{b}\right)+\left(\nabla^{c} \xi^{b}\right)\left(\nabla_{c} \xi_{b}\right)\right] d V=0 .
$$

Since (2.1) is an isometry, we have

$$
\nabla_{c} \xi_{b}+\nabla_{b} \xi_{c}=2 h_{c b x} \xi^{x}, \quad \nabla_{a} \xi^{a}=h_{c}{ }^{c} \xi^{x}
$$

Thus substituting these into the above equation, we find

$$
\int\left[-K_{c b} \xi^{c} \xi^{b}-2\left(h^{c b}{ }_{y} h_{c b x}\right) \xi^{y} \xi^{x}+\left(h_{c}^{c} x \xi^{x}\right)^{2}+\left(\nabla^{c} \xi^{b}\right)\left(\nabla_{c} \xi_{b}\right)\right] d V=0 .
$$

From (3.2), we have 
THEOREM 3.1. If an infinitesimal isometric variation of a compact orientable submanifold $M^{n}$ of a Riemannian manifold $M^{m}$ satisfies

$$
K_{c b} \xi^{c} \xi^{b}+2\left(h_{y}^{c b} h_{c b x}\right) \xi^{y} \xi^{x} \leqq 0,
$$

then $\xi^{a}$ satisfies

$$
\nabla_{c} \xi^{a}=0 \text { and cosequently } K_{c b} \xi^{c} \xi^{b}=0
$$

and

$$
h_{c b x} \xi^{x}=0 \text {, }
$$

that is, $M^{n}$ is geodesic in the direction $\xi^{x}$. Moreover if $M^{n}$ is irreducible, then $\xi^{a}=0$, that is, the variation is normal and the submanifold is geodesic in the direction of the variation.

\section{$\S 4$. Infinitesimal affine variations.}

For an infinitesimal affine variation (2.1), we have (2.14), from which, by transvection with $g^{c b}$, we obtain

$$
g^{c b} \nabla_{c} \nabla_{b} \xi^{a}+K_{d}{ }^{a} \xi^{d}-2 \nabla^{c}\left(h_{c}{ }^{a}{ }_{x} \xi^{x}\right)+\nabla^{a}\left(h_{c}{ }^{c} \xi^{x}\right)=0
$$

and, by contraction with respect to $a$ and $b$, we have

$$
\nabla_{c}\left(\nabla_{a} \xi^{a}-h_{a}{ }^{a}{ }_{x} \xi^{x}\right)=0
$$

and consequently we obtain

$$
\nabla_{a} \xi^{a}-h_{a}{ }^{a}{ }_{x} \xi^{x}=\text { constant } .
$$

Thus (2.16) becomes

$$
\begin{array}{r}
\int\left[\frac{1}{2}\left(\nabla_{c} \xi_{b}+\nabla_{b} \xi_{c}-2 h_{c b y} \xi^{y}\right)\left(\nabla^{c} \xi^{b}+\nabla^{b} \xi^{c}-2 h^{c b} \xi_{x}\right)\right. \\
\left.+\left(\nabla_{c} \xi_{b}+\nabla_{b} \xi_{c}-2 h_{c b y} \xi^{y}\right) h^{c b} \xi_{x} \xi^{x}\right] d V=0
\end{array}
$$

because of $\int\left(\nabla_{b} \xi^{b}\right) d V=0$.

Thus if

$$
\left(\nabla_{c} \xi_{b}+\nabla_{b} \xi_{c}-2 h_{c b y} \xi^{y}\right) h^{c b} \xi^{x}=0 .
$$

then we have, from the equation above,

$$
\nabla_{c} \xi_{b}+\nabla_{b} \xi_{c}-2 h_{c b y} \xi^{y}=0 .
$$

The converse being evident, we have 
THEOREM 4.1. If an infinitesimal affine variation of a compact orientable submanifold of a Riemannian manifold satisfies (4.4) then the variation is an isometry.

\section{§5. Infinitesimal conformal variations.}

If an infinitesimal variation (2.1) is conformal, we have

$$
\nabla_{c} \xi_{b}+\nabla_{b} \xi_{c}-2 h_{c b x} \xi^{x}=2 \lambda g_{c b}
$$

for a certain function $\lambda$, from which, transvecting with $g^{c b}$, we find

$$
\lambda=\frac{1}{n}\left(\nabla_{a} \xi^{a}-h_{a}^{a}{ }_{x} \xi^{x}\right) .
$$

Thus we can write (5.1) as

$$
\nabla_{c} \xi_{b}+\nabla_{b} \xi_{c}-2 h_{c b x} \xi^{x}-\frac{2}{n}\left(\nabla_{e} \xi^{e}-h_{e}^{e}{ }_{x} \xi^{x}\right) g_{c b}=0 .
$$

On the other hand, for an infinitesimal conformal variation, we have $\delta g_{c b}=2 \lambda g_{c b} \varepsilon$ and consequently from (2.12),

$$
\delta \Gamma_{c b}^{a}=\left(\delta_{c}^{a} \lambda_{b}+\delta_{b}^{a} \lambda_{c}-g_{c b} \lambda^{a}\right) \varepsilon,
$$

where $\lambda_{b}=\nabla_{b} \lambda$ and $\lambda^{a}=\nabla^{a} \lambda$, that is,

$$
\begin{aligned}
\nabla_{c} \nabla_{b} \xi^{a}+ & K_{d c b}{ }^{a} \xi^{d}-\nabla_{c}\left(h_{b}{ }^{a}{ }_{x} \xi^{x}\right)-\nabla_{b}\left(h_{c}{ }^{a}{ }_{x} \xi^{x}\right)+\nabla^{a}\left(h_{c b x} \xi^{x}\right) \\
& =\delta_{c}^{a} \lambda_{b}+\delta_{b}^{a} \lambda_{c}-g_{c b} \lambda^{a},
\end{aligned}
$$

from which, transvecting with $g^{c b}$, we find

$$
g^{c b} \nabla_{c} \nabla_{b} \xi^{a}+K_{d}{ }^{a} \xi^{d}-2 \nabla^{c}\left(h_{c}{ }^{a}{ }_{x} \xi^{x}\right)+\nabla^{a}\left(h_{c}{ }^{c} \xi^{x}\right)=-(n-2) \lambda^{a},
$$

or, $\lambda$ being given by (5.2),

$$
\begin{gathered}
g^{c b} \nabla_{c} \nabla_{b} \xi^{a}+K_{d}{ }^{a} \xi^{d}-2 \nabla^{c}\left(h_{c}{ }^{a}{ }_{x} \xi^{x}\right)+\nabla^{a}\left(h_{c}{ }^{c} \xi_{x} \xi^{x}\right) \\
+\frac{n-2}{n} \nabla^{a}\left(\nabla_{e} \xi^{e}-h_{e}{ }^{e} \xi^{x}\right)=0 .
\end{gathered}
$$

Now, we can transform (2.16) into

$$
\begin{gathered}
\int\left[\left\{g^{c b} \nabla_{c} \nabla_{b} \xi^{a}+K_{d}{ }^{a} \xi^{d}-2 \nabla^{c}\left(h_{c}{ }^{a}{ }_{x} \xi^{x}\right)+\nabla^{a}\left(h_{c}{ }^{c}{ }_{x} \xi^{x}\right)\right.\right. \\
\left.\div \frac{n-2}{n} \nabla^{a}\left(\nabla_{e} \xi^{e}-h_{e}{ }^{e} \xi_{x} \xi^{x}\right)\right\} \xi_{a}
\end{gathered}
$$




$$
\begin{aligned}
& +\frac{1}{2}\left\{\nabla_{c} \xi_{b}+\nabla_{b} \xi_{c}-2 h_{c b y} \xi^{y}-\frac{2}{n}\left(\nabla_{e} \xi^{e}-h_{e}{ }_{x} \xi^{x}\right) g_{c b}\right\} \\
& \quad \times\left\{\nabla^{c} \xi^{b}+\nabla^{b} \xi^{c}-2 h^{c b}{ }_{x} \xi^{x}-\frac{2}{n}\left(\nabla_{d} \xi^{d}-h_{d}{ }^{d}{ }_{x} \xi^{x}\right) g^{c b}\right\} \\
& \left.+\left\{\nabla_{c} \xi_{b}+\nabla_{b} \xi_{c}-2 h_{c b y} \xi^{y}-\frac{2}{n}\left(\nabla_{e} \xi^{e}-h_{e}{ }_{y} \xi^{y}\right) g_{c b}\right\} h^{c b}{ }_{x} \xi^{x}\right] d V=0 .
\end{aligned}
$$

Thus if the infinitesimal variation is conformal, we have (5.3) and consequently

$$
\left\{\nabla_{c} \xi_{b}+\nabla_{b} \xi_{c}-2 h_{c b y} \xi^{y}-\frac{2}{n}\left(\nabla_{e} \xi^{e}-h_{e}^{e}{ }_{y} \xi^{y}\right) g_{c b}\right\} h^{c b}{ }_{x} \xi^{x}=0
$$

and also (5.6),

Conversely if (5.6) and (5.8) are satisfied, we have from (5.7)

$$
\nabla_{c} \xi_{b}+\nabla_{b} \xi_{c}-2 h_{c b y} \xi^{y}-\frac{2}{n}\left(\nabla_{e} \xi^{e}-h_{e}{ }^{e} \xi^{y}\right) g_{c b}=0,
$$

which shows that the infinitesimal variation is conformal. Thus we have

THEOREM. 5.1. In order for an infinitesimal variation (2.1) to be conformal, it is necessary and sufficient that (5.6) and (5.8) hold.

Substituting (5.6) into (3.1), we find

$$
\begin{aligned}
\frac{1}{2} \Delta\left(\xi^{a} \xi_{a}\right)= & -K_{c b} \xi^{c} \xi^{b}+2 \xi_{a} \nabla^{c}\left(h_{c}{ }^{a}{ }_{x} \xi^{x}\right)-\xi_{a} \nabla^{a}\left(h_{c}{ }^{c}{ }_{x} \xi^{x}\right) \\
& -\frac{n-2}{n} \xi_{a} \nabla^{a}\left(\nabla_{e} \xi^{e}-h_{e}{ }^{e} \xi^{x}\right)+\left(\nabla^{c} \xi^{b}\right)\left(\nabla_{c} \xi_{b}\right) .
\end{aligned}
$$

from which, integrating over $M^{n}$,

$$
\begin{aligned}
& \int\left[-K_{c b} \xi^{c} \xi^{b}-\left(h^{c b}{ }_{x} \xi^{x}\right)\left(\nabla_{c} \xi_{b}+\nabla_{b} \xi_{c}\right)+h_{c}{ }^{c}{ }_{x} \xi^{x}\left(\nabla_{a} \xi^{a}\right)\right. \\
& \left.+\frac{n-2}{n}\left(\nabla_{e} \xi^{e}-h_{e}{ }^{e} \xi^{x}\right)\left(\nabla_{a} \xi^{a}\right)+\left(\nabla^{c} \xi^{b}\right)\left(\nabla_{c} \xi_{b}\right)\right] d V=0 .
\end{aligned}
$$

Since the variation is conformal, we have

$$
\nabla_{c} \xi_{b}+\nabla_{b} \xi_{c}=2 h_{c b x} \xi^{x}+\frac{2}{n}\left(\nabla_{e} \xi^{e}-h_{e}^{e}{ }_{x} \xi^{x}\right) g_{c b} .
$$

Substituting this into the above integral formula, we find

$$
\begin{gathered}
\int\left[-K_{c b} \xi^{c} \xi^{b}-2 h^{c b}{ }_{y} \xi^{y} h_{c b x} \xi^{x}-\frac{2}{n} h_{c}{ }^{c} \xi_{y}^{y}\left(\nabla_{e} \xi^{e}-h_{e}{ }_{x} \xi^{x}\right)+h_{c}{ }^{c}{ }_{x} \xi^{x}\left(\nabla_{a} \xi^{a}\right)\right. \\
\left.+\frac{n-2}{n}\left(\nabla_{e} \xi^{e}-h_{e}{ }^{e} \xi^{x}\right)\left(\nabla_{a} \xi^{a}\right)+\left(\nabla^{c} \xi^{b}\right)\left(\nabla_{c} \xi_{b}\right)\right] d V=0
\end{gathered}
$$


from which

$$
\begin{gathered}
\int\left[-K_{c b} \xi^{c} \xi^{b}-2\left(h_{y}^{c b}-\frac{1}{n} g^{c b} h_{e}{ }^{e} y\right)\left(h_{c b x}-\frac{1}{n} g_{c b} h_{d}{ }^{d}\right) \xi^{y} \xi^{x}\right. \\
\left.+\frac{n-2}{n}\left(\nabla_{a} \xi^{a}\right)^{2}+\left(\nabla^{c} \xi^{b}\right)\left(\nabla_{c} \xi_{b}\right)\right] d V=0 .
\end{gathered}
$$

Thus from (5.9), we have

THEOREM 5.2. If an infinitesimal conformal variation of a compact orientable submanifold $M^{n}$ of a Riemannian manifold $M^{m}$ satisfies

$$
K_{c b} \xi^{c} \xi^{b}+2\left(h_{y}^{c b}-\frac{1}{n} g^{c b} h_{e y}^{e}\right)\left(h_{c b x}-\frac{1}{n} g_{c b} h_{d}{ }^{d}\right) \xi^{y} \xi^{x} \leqq 0,
$$

then, $\xi^{a}$ satisfies

$$
\nabla_{c} \xi^{a}=0 \text { and consequently } K_{c b} \xi^{c} \xi^{b}=0
$$

and

$$
h_{c b x} \xi^{x}=\frac{1}{n} g_{c b} h_{d}{ }^{d} \xi^{x},
$$

that is, $M^{n}$ is umbilical in the direction $\xi^{x}$. Moreover if $M^{n}$ is irreducible, then $\xi^{a}=0$, that is, the variation is normal and the submanifold is umbilical in the direction of the variation.

\section{Bibliography}

[1] Bang-yen Chen, Geometry of Submanifolds, Marcel Dekker, Inc., 1975.

[2] J.A. Schouten, Ricci-Calculus, Springer-Verlag, 1954.

[3] K. Yano, Sur la théorie des déformations infinitésimales, J. Fac. Sci. Univ. Tokyo, 6 (1949), 1-75.

[4] K. Yano, Integral formulas in Riemannian geometry, Marcel Dekker, Inc., 1970.

[5] K. Yano, Infinitesimal variations of submanifolds, Kodai Math. J., 1 (1978), 30-44.

\section{Kentaro YANO}

Tokyo Institute of Technology Meguro-ku, Tokyo 152 Japan 